Research Article

\title{
Distinctive Alterations of Functional Connectivity Strength between Vascular and Amnestic Mild Cognitive Impairment
}

\author{
Hui Li $\left(\mathbb{D},{ }^{1}\right.$ Shuai Gao, ${ }^{1}$ Xiuqin Jia, ${ }^{1}$ Tao Jiang $\mathbb{D},{ }^{1}$ and Kuncheng Li $\mathbb{D}^{2,3}$ \\ ${ }^{1}$ Department of Radiology, Beijing Chaoyang Hospital, Capital Medical University, Beijing 100020, China \\ ${ }^{2}$ Department of Radiology, Xuanwu Hospital, Capital Medical University, Beijing 10053, China \\ ${ }^{3}$ Beijing Key Lab of MRI and Brain Informatics, Beijing 10053, China
}

Correspondence should be addressed to Tao Jiang; jiangt8166@hotmail.com and Kuncheng Li; cjr.likuncheng@vip.163.com

Received 27 May 2020; Revised 2 November 2020; Accepted 30 April 2021; Published 20 May 2021

Academic Editor: Clive.R. Bramham

Copyright (C) 2021 Hui Li et al. This is an open access article distributed under the Creative Commons Attribution License, which permits unrestricted use, distribution, and reproduction in any medium, provided the original work is properly cited.

\begin{abstract}
Widespread structural and functional alterations have been reported in the two highly prevalent mild cognitive impairment (MCI) subtypes, amnestic MCI (aMCI) and vascular MCI (VaMCI). However, the changing pattern in functional connectivity strength (FCS) remains largely unclear. The aim of the present study is to detect the differences of FCS and to further explore the detailed resting-state functional connectivity (FC) alterations among VaMCI subjects, aMCI subjects, and healthy controls (HC). Twenty-six aMCI subjects, $31 \mathrm{VaMCI}$ participants, and $36 \mathrm{HC}$ participants underwent cognitive assessments and resting-state functional MRI scans. At first, one-way ANCOVA and post hoc analysis indicated significant decreased FCS in the left middle temporal gyrus (MTG) in aMCI and VaMCI groups compared to HC, especially in the VaMCI group. Then, we selected the left MTG as a seed to further explore the detailed resting-state FC alterations among the three groups, and the results indicated that FC between the left MTG and some frontal brain regions were significantly decreased mainly in VaMCI. Finally, partial correlation analysis revealed that the FC values between the left MTG and left inferior frontal gyrus were positively correlated with the cognitive performance episodic memory and negatively related to the living status. The present study demonstrated that different FCS alterations existed in aMCI and VaMCI. These findings may provide a novel insight into the understanding of pathophysiological mechanisms underlying different MCI subtypes.
\end{abstract}

\section{Introduction}

Mild cognitive impairment (MCI), associated with deficits in multiple cognitive domains without notable affection on daily activities, is regarded as a risk state for dementia [1]. The two highly prevalent subtypes, amnestic MCI (aMCI) and vascular MCI (VaMCI), are considered the prodromal stage of Alzheimer's disease (AD) and vascular dementia (VD), respectively $[2,3]$. It has been a notion that VaMCI subjects exhibit significant impairment in executive function and semantic memory while aMCI subjects show predominant deficits in episodic memory [4].

Magnetic resonance imaging (MRI) is an important imaging method for pathophysiological mechanism investigation and differential diagnosis of MCI subtypes. Wide- spread structural abnormalities and functional alterations have been reported in both VaMCI and aMCI groups [58]. Gray matter atrophies are mainly distributed in frontal and temporal brain regions, posteromedial cortices, and several subcortical brain sites, especially the medial temporal lobe, including the hippocampus and entorhinal cortex [9]. Using diffusion MRI scanning, microstructural deteriorations within the corpus callosum, capsule, periventricular white matter, cingulum, and occipitofrontal fasciculi have been reported [10-12]. Yu et al. reported significant differences in the relationship between fractional anisotropy (FA) and the Auditory Verbal Learning Test (AVLT) between the VaMCI and aMCI groups [13]. Functional connectivity (FC) changes are predominantly found in the default mode network and the medial temporal lobe $[5,6,14]$. Both 
decreased and increased brain activities related to the severity of cognitive decline have been demonstrated. Graph theory-based network analyses also reveal significant dysregulation of the topological organization of functional brain networks [15].

Recently, based on the graph theory, the human brain is considered a complex, interconnected network with a set of nodes linked by connections to support efficient information processing and integration [16]. Converging evidences in both structural [17] and functional network analyses [18] have demonstrated that some specific nodes with a large number of connections (or large degree) [19] play critical roles in fast information integration and communication with minimal energy cost [20]. Previous studies have investigated the changing pattern of functional network centrality by calculating functional connectivity strength (FCS) in some diseases such as social anxiety disorder and provide a novel insight into the understanding of underlying pathophysiological mechanisms [21]. Although previous studies have demonstrated altered FC in different MCI subtypes, the changing pattern in FCS remains unclear.

In the present study, we detected the differences in FCS among VaMCI subjects, aMCI subjects, and healthy control (HC) participants. Then, seed-based connectivity analysis were performed to further explore the detailed resting-state FC alterations, using the clusters showing significant differences in FCS as the seeds. We assumed that these MCI subtypes would show distinct FCS and FC changes within their signature large-scale networks as compared to HC. Further, we would explore how these changes relate to neuropsychological deficits in VaMCI and aMCI.

\section{Materials and Methods}

2.1. Participants. The study was conducted under a research protocol approved by the Ethics Committee of Beijing Xuanwu Hospital. Written informed consent was obtained from all participants prior to the study.

Twenty-six aMCI subjects, 31 VaMCI subjects, and 36 HC participants were recruited from Xuanwu Hospital in Beijing between 2017 and 2019. We followed the methods of Li et al. [22]. All participants underwent detailed medical history collection, physical examination, and neuropsychological evaluation by experienced neurologists that have been trained to unify the evaluation criteria. Neuropsychological assessments included Clinical Dementia Rating (CDR), Mini Mental State Examination (MMSE), Montreal Cognitive Assessment (MoCA), and activities of daily living (ADL).

The diagnosis of aMCI followed the criteria stipulated in 2011 by the National Institute on Aging and the Alzheimer's Association [3]. Other two conditions were satisfied as follows: (a) CDR score of 0.5 and a score of at least 0.5 on the memory domain [23] and (b) medial temporal lobe or hippocampal atrophy on MRI $[24,25]$.

The criteria used for the selection for the $\mathrm{HC}$ were as follows: (a) no complaints of cognitive changes, (b) no current or previous diagnosis of any neurological or psychiatric disorders, (c) no neurological deficiencies in physical examina- tions, (d) no abnormal neurological findings on brain MRI, and (e) CDR score of 0 .

The inclusion criteria for the VaMCI were based on the Diagnostic and Statistical Manual of Mental Disorders (DSM), fourth edition, for VaMCI [26] including the following: (a) complaint of cognitive impairment, at least at one cognitive domain; (b) objective evidence for cognitive decline; (c) the clinical characteristics being consistent with the vascular etiology; (d) history of cerebrovascular disease, physical examination, and/or neuroimaging evidence for cerebrovascular disease; (c) exclusion of other possible diseases; and (d) ability to maintain independence in daily activities.

Participants were excluded if they had a history of psychiatric disorder and neurological conditions affecting cognition, such as head injury, depression, alcohol use disorder, epilepsy, and Parkinson's disease. Additional exclusion criteria included major medical illness, severe visual or hearing loss, and contraindications for MRI.

2.2. Magnetic Resonance Imaging Procedures. The subjects were scanned using a specific 3-Tesla GE scanner (General Electric, MRI750W, America). All participants were asked to remain still, stay awake, and keep their eyes closed. Head motion and scanner noise were reduced using foam padding and earplugs. All subjects underwent clinical standardized axial T2, axial fluid-attenuated inversion recovery (FLAIR), sagittal T1, and resting-state funcional MRI(fMRI)scans using an echo-planar imaging (EPI) sequence. The following parameters were used for fMRI images for aMCI and $\mathrm{HC}$ participants: repetition time $(\mathrm{TR})=2000 \mathrm{~ms}$, echo time $(\mathrm{TE})$ $=30 \mathrm{~ms}$, flip angle $(\mathrm{FA})=90^{\circ}$, field of view $(\mathrm{FOV})=256 \times$ $256 \mathrm{~mm}^{2}$, data matrix $=64 \times 64,36$ axial slices, slice thickness/gap $=3 / 1 \mathrm{~mm}$, and number of repetitions $=180$. Meanwhile, the following parameters were used for fMRI images for VaMCI subjects: $\mathrm{TR}=2000 \mathrm{~ms}$, TE $=30 \mathrm{~ms}$, FA $=90^{\circ}, \mathrm{FOV}=220 \times 220 \mathrm{~mm}^{2}$, slice thickness $/$ gap $=3.6 / 0.4$ $\mathrm{mm}, 36$ axial slices, data matrix $=64 \times 64$, and number of repetitions $=185$. Parameters for axial T2 were as follows: $\mathrm{TR}=4581 \mathrm{~ms}, \quad \mathrm{TE}=82 \mathrm{~ms}, \quad \mathrm{FOV}=220 \times 220 \mathrm{~mm}^{2}$, slice thickness $/$ gap $=5.5 / 1.0 \mathrm{~mm}$, data matrix $=416 \times 416$, and 20 axial slices. Parameters for sagittal T1 were as follows: TR = $1750 \mathrm{~ms}, \mathrm{TE}=24 \mathrm{~ms}, \mathrm{FOV}=240 \times 216 \mathrm{~mm}^{2}$, slice thickness $/$ gap $=5.5 / 1.0 \mathrm{~mm}$, data matrix $=288 \times 224$, and 20 axial slices. Parameters for axial FLAIR were as follows: TR = $7000 \mathrm{~ms}, \quad \mathrm{TE}=120 \mathrm{~ms}, \quad \mathrm{FOV}=220 \times 220 \mathrm{~mm}^{2}$, slice thickness $/$ gap $=5.5 / 1.0 \mathrm{~mm}$, data matrix $=416 \times 416$, and 20 axial slices.

2.3. MRI Data Preprocessing. Functional image data were preprocessed using the Data Processing Assistant for Resting-State fMRI (DPARSF) software package v4.5 [27] and Statistical Parametric Mapping 12 based on MATLAB 2013a. The first 5 images of each fMRI dataset were discarded to reduce the initial fluctuation of MRI signals in aMCI and $\mathrm{HC}$, while the first 10 images of each fMRI dataset were discarded in VaMCI. The numbers of remaining images were all the same (175 images) in the three groups. Briefly, the fMRI time series were first corrected for within-scan acquisition 
time differences between slices and realigned to correct for head motion. The participants with head movement exceeding $2.0 \mathrm{~mm}$ of translation or $2.0^{\circ}$ of rotation in any direction were excluded. In addition, one-way analysis of variance showed that there was no significant difference in the values of the mean framewise displacement (FD) among the three groups. All the realigned images were spatially normalized to the Montreal Neurological Institute EPI template, and each voxel was resampled to $3 \times 3 \times 3 \mathrm{~mm}^{3}$. To avoid introducing artificial local spatial correlations, the images were not smoothed [28]. Denoising steps included linear detrending and regression of the six motion parameters and their first-order derivatives and regression of white matter and cerebrospinal fluid (CSF) [29]. Then, the time series were temporally band-pass filtered $(0.01-0.08 \mathrm{~Hz})$ to reduce the effects of high-frequency physiological noises.

2.4. Network Analysis. For each voxel, the time series was extracted and Pearson's correlation coefficients between the time series of the voxel and all other voxels' time series were calculated within a gray matter mask $(N$ voxels $=58,108)$. Then, the correlation coefficients greater than 0.2 were averaged over the gray matter mask, and a 3D FCS map for each subject was obtained [30]. Finally, the FCS map was converted to $z$ scores and smoothed with a $6 \mathrm{~mm}$ full width at half-maximum Gaussian kernel.

\section{Statistical Analyses}

3.1. Demographic and Neuropsychological Variables. For gender assessment, $x^{2}$ tests were used. The age, education level, and cognitive performance differences among the three groups were estimated by one-way analysis of variance. These analyses were implemented in SPSS 21.

3.2. Group Differences in Functional Connectivity Strength. One-sample $t$-tests were performed to identify the patterns of FCS within each group [21], and the significant threshold was set at voxel-level $p<0.05$ corrected for multiple comparisons using the family-wise error rate (FWE). To find the altered FCS regions, one-way analysis of covariance (ANCOVA) was then performed to compare the FCS maps among the three groups, with age, gender, and education as covariates. The significant threshold was set at cluster-level $p<0.05$, FWE corrected. The FCS values of the regions showing significant group difference were extracted for post hoc pairwise comparisons and partial correlations. Post hoc pairwise comparisons were conducted by independentsample $t$-tests to compute the differences between any two groups using SPSS 21.

3.3. Seed-Based Resting-State Functional Connectivity Analysis. Seed-based connectivity analysis were performed to further explore the detailed resting-state FC alterations, using the clusters showing significant differences in FCS as the seeds. One-way ANCOVA was performed on the FC maps for each identified seed. The significant threshold was set at voxel-level $p<0.05$, FWE corrected, with age, gender, and years of education as covariates. The FC values of the regions showing significant group differences were extracted for post hoc pairwise comparisons and partial correlations.

3.4. Relationships between Regional Connectivity Measures and Cognitive Performance. Partial correlation analysis was performed to explore the relationship between the connectivity measurements (i.e., FCS and seed-based FC) and clinical variables in VaMCI and aMCI separately, with age, gender, and years of education as covariates. Statistical significance was set at $p<0.05$.

\section{Results}

4.1. Demographics and Clinical Characteristics of the Participants. The demographic and clinical data are shown in Table 1. Significant differences were found in cognitive assessments among the three groups while age, gender, and education years were matched well. Both MCI subtype groups showed lower scores in MMSE $(p<0.001)$ and MoCA $(p<0.001)$ and higher CDR scores $(p<0.001)$, indicating significant general cognitive decline as compared to HC. Meanwhile, both MCI subtype groups showed higher ADL scores $(p<0.001)$. In addition, the aMCI group showed lower cognitive assessment scores and higher ADL scores than VaMCI.

4.2. Within-Group FCS Analyses. The results derived from one-sample $t$-tests for the three groups are separately shown in Figures S1-S3. Functional nodes with a large number of connections were found mainly in the temporal and parietal brain regions, as well as several occipital and frontal cortices.

4.3. Between-Group FCS Analyses. One-way ANCOVA indicated significant FCS difference in the left middle temporal gyrus (MTG) among the three groups (Table 2, Figure 1).

Post hoc analysis revealed that (1) as compared to HC, subjects with aMCI showed significant decreased FCS in the left MTG; (2) as compared to HC, subjects with VaMCI showed significant decreased FCS in the left MTG; and (3) as compared to aMCI, subjects with VaMCI showed significant decreased FCS in the left MTG (Figure 1).

4.4. Alterations in Seed-Based Resting-State Functional Connectivity. For further detailed analysis regarding the left MTG FC pattern, the subsequent seed-based FC analysis revealed that the left MTG network in HC was composed of the medial frontal, temporal cortical, and parietal sites. However, the frontal sites were excluded in both aMCI and VaMCI (Figures S4-S6). One-way ANCOVA indicated significant FC differences between the left MTG and the right orbital frontal gyrus (OFG), the left inferior frontal gyrus (IFG), and the right IFG among the three groups. Post hoc analysis revealed that significant FC disruptions in the right OFG and the left IFG were found only in VaMCI, while significant reduced FC in the right IFG were found in both aMCI and VaMCI with more serious disruption in the VaMCI group (Table 2, Figure 2). 
TABLE 1: Clinical characteristics of subjects with aMCI and VaMCI and HC.

\begin{tabular}{|c|c|c|c|c|}
\hline Characteristics & $\mathrm{HC}(n=36)$ & $\operatorname{VaMCI}(n=31)$ & aMCI $(n=26)$ & Group $p$ \\
\hline Age (years) & $64.22 \pm 6.97$ & $64.93 \pm 10.11$ & $66.04 \pm 7.92$ & 0.62 \\
\hline Gender, M/F & $17 / 19$ & $18 / 13$ & $8 / 18$ & 0.11 \\
\hline Education (years) & $10.72 \pm 5.41$ & $9.00 \pm 2.00$ & $10.77 \pm 4.65$ & 0.08 \\
\hline CDR & 0 & 0.5 & 0.5 & $<0.001$ \\
\hline MMSR & $28.13 \pm 2.77$ & $26.32 \pm 2.05$ & $23.73 \pm 3.85$ & $<0.001$ \\
\hline MoCA & $25.22 \pm 2.89$ & $23.32 \pm 1.32$ & $19.81 \pm 4.71$ & $<0.001$ \\
\hline $\mathrm{ADL}$ & $20.00 \pm 0.00$ & $20.87 \pm 1.43$ & $22.84 \pm 2.78$ & $<0.001$ \\
\hline
\end{tabular}

Values represent means $\pm \mathrm{SD}$; $p$ values were derived from the one-way NOVA test comparing the three groups, except for "gender" where the $p$ value was obtained using the $x^{2}$ test. MMSE: Mini Mental State Examination; MoCA: Montreal Cognitive Assessment; ADL: activities of daily living; VaMCI: vascular mild cognitive impairment; aMCI: amnestic mild cognitive impairment; HC: healthy control.

TABLE 2: FCS differences and left MTG FC differences among subjects with aMCI and VaMCI and HC.

\begin{tabular}{lccccc}
\hline Brain regions & Cluster size & $x$ & $y$ & $z$ & F-score \\
\hline FCS & 189 & -60 & -3 & -21 & 9.823 \\
Lt. MTG & & & & & \\
FC & 26 & 33 & 48 & -3 & 20.8862 \\
Rt. OFG & 50 & 57 & 33 & 6 & 27.3006 \\
Rt. IFG & 98 & -45 & 18 & 18 & 27.7231 \\
Lt. IFG &
\end{tabular}

Lt.: left; Rt.: right; FC: functional connectivity; MTG: middle temporal gyrus; OFG: orbital frontal gyrus; IFG: inferior frontal gyrus; VaMCI: vascular mild cognitive impairment; aMCI: amnestic mild cognitive impairment; HC: healthy control; MNI: Montreal Neurological Institute.

4.5. Correlations between Cognitive Performances and Connectivity Measures. Partial correlation analysis revealed that the FC values between the left MTG and left IFG were positively correlated with the cognitive performance episodic memory measured by MoCA scores $(r=0.41, p=0.03$, Figure 3(a)) and negatively correlated with ADL scores $(r=-0.44, p=0.02$, Figure 3(b) $)$ in the VaMCI group.

\section{Discussion}

Based on resting-state fMRI and graph theory approaches, the present study reported decreased FCS in the left MTG in both VaMCI subjects and aMCI subjects, especially in the VaMCI group. Furthermore, more frontal regions showed disrupted resting-state FC to left MTG in the VaMCI group than in the aMCI group. These aberrant brain connectivities may be the neurobiological mechanism underlying the cognitive deficits.

Based on the computational analysis of anatomic connectivity, Sporns et al. formally defined the cortex nodes that have disproportionately numerous connections as hubs [31]. Network analysis studies using postmortem tracing techniques in nonhuman primates [32], vivo tract-tracing [33], and fMRI in humans [34] further confirmed the existence of hubs. An accurate reference map of prominent cortical hubs consisting of posterior cingulate, lateral temporal,

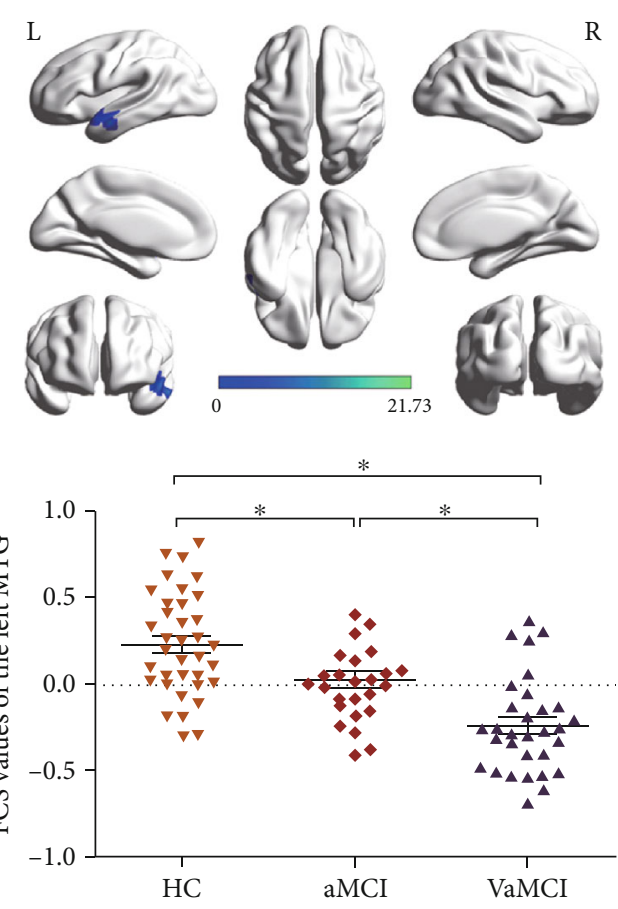

FIgURE 1: The result of one-way ANCOVA and post hoc analysis indicated significant decreased FCS in the left MTG among the VaMCI, aMCI, and HC. $*$ means $p<0.05$ in the two-sample $t$-test using SPSS21. L: left; R: right; MTG: middle temporal gyrus; FCS: functional connectivity strength; VaMCI: vascular mild cognitive impairment; aMCI: amnestic mild cognitive impairment; HC: healthy control.

lateral parietal, and medial/lateral prefrontal cortices had been obtained, and the left MTG is one of the peak locations of the largest 10 hubs [35].

MTG plays an important role in verbal short-term memory [36] and global cognitive function [37]. Previous studies have shown that MTG is one of the most vulnerable brain regions affected by cognition impairment-related pathological changes. In voxel-based morphometric study, significant gray matter volume reductions in MTG have been reported in both patients with aMCI and VaMCI [38-40]. As for the whole-brain function network, an altered FC pattern and FC density in MTG have been reported by fMRI $[5,41,42]$. 


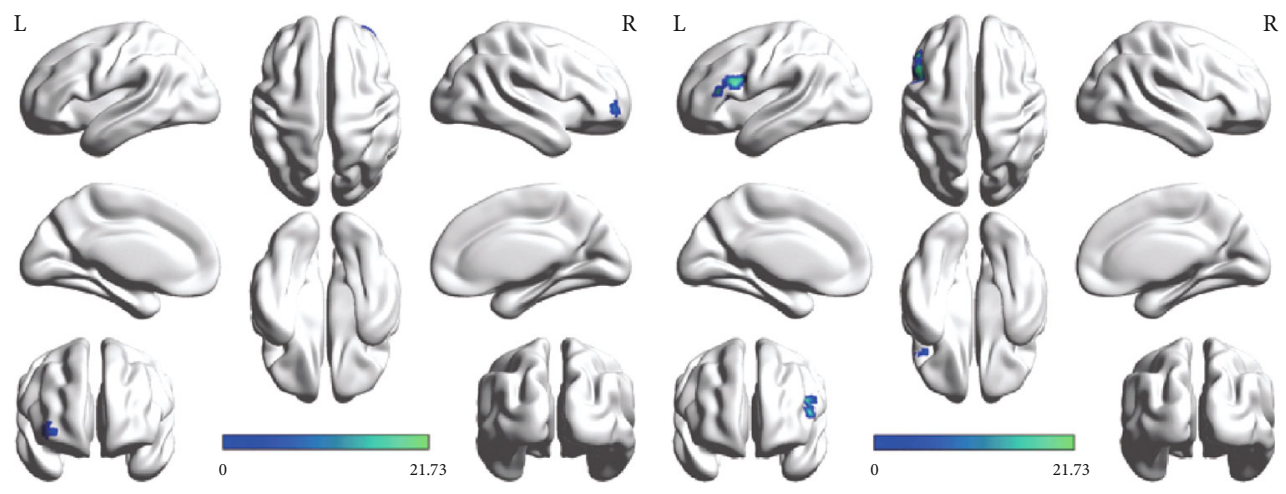

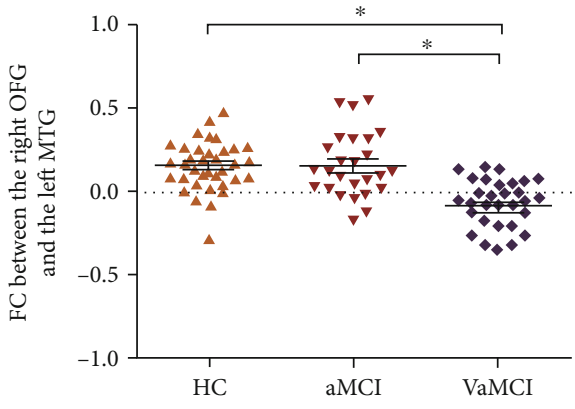

(a)

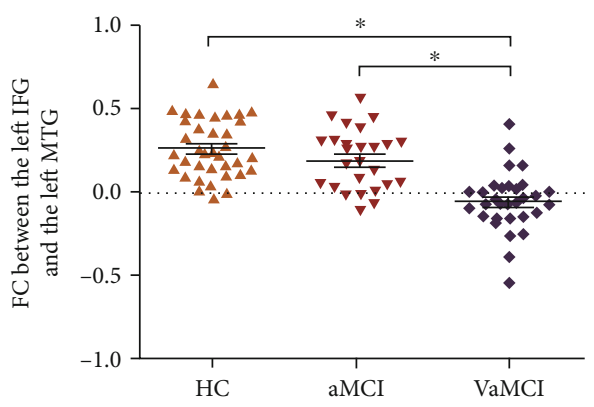

(b)
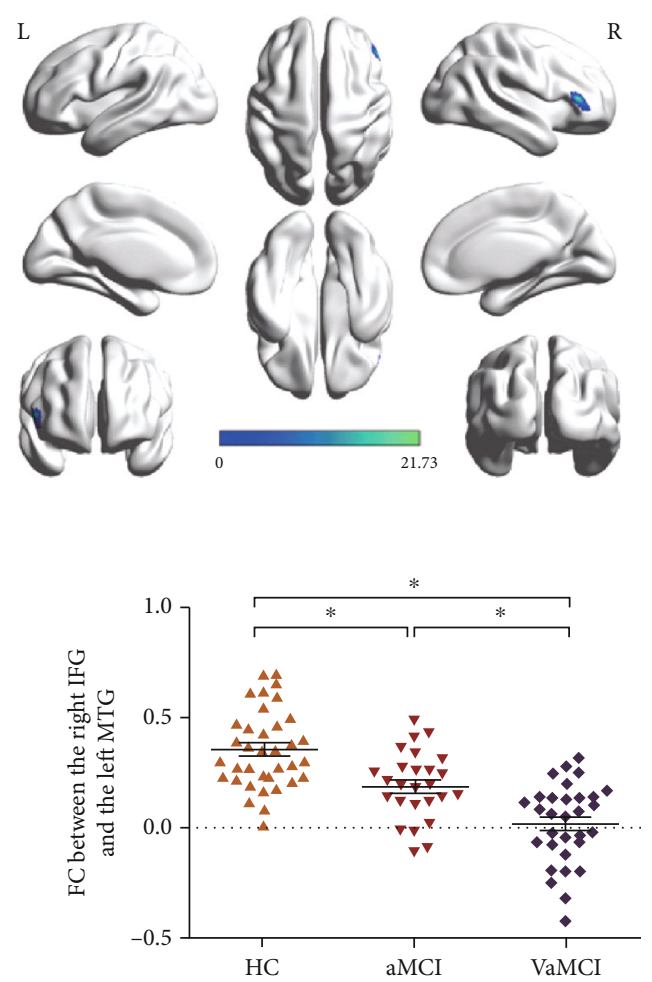

(c)

FIGURE 2: The results of one-way ANCOVA and post hoc analysis indicated significant decreased FC between the left MTG and the right OFG (a), the left IFG (b), and the right IFG (c) among the three groups. * means $p<0.05$ in the two-sample $t$-test using SPSS21. L: left; R: right; FC: functional connectivity; MTG: middle temporal gyrus; OFG: orbital frontal gyrus; IFG: inferior frontal gyrus; VaMCI: vascular mild cognitive impairment; aMCI: amnestic mild cognitive impairment; HC: healthy control. 


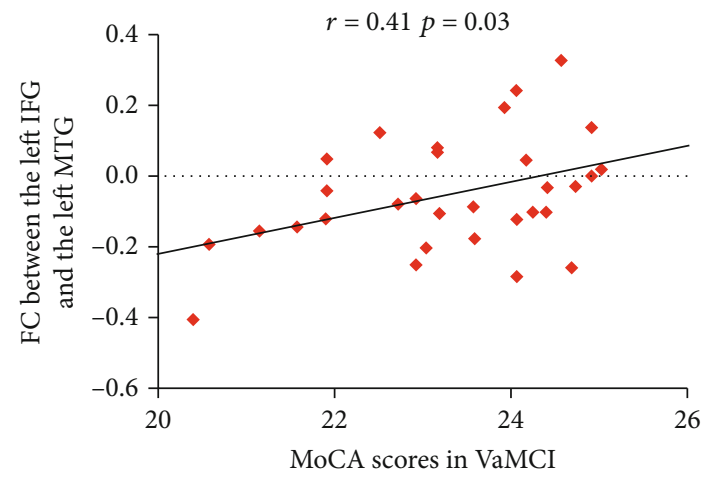

(a)

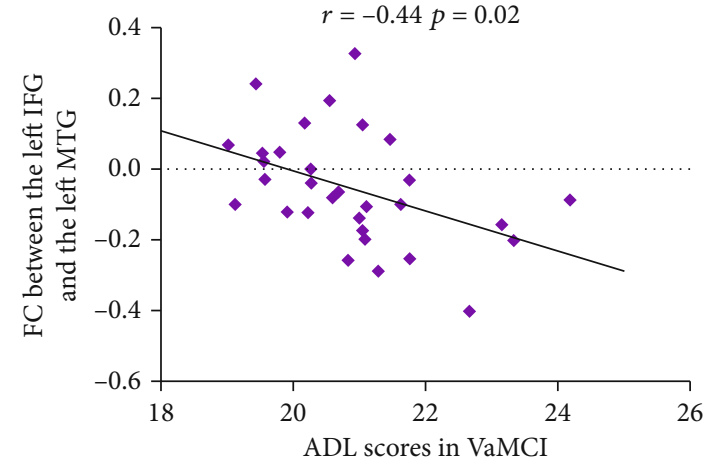

(b)

FIGURE 3: (a) The FC values between the left MTG and left IFG were positively correlated with the cognitive performance episodic memory measured by MoCA scores. (b) The FC values between the left MTG and left IFG were negatively correlated with ADL scores. FC: functional connectivity; MTG: middle temporal gyrus; IFG: inferior frontal gyrus; MoCA: Montreal Cognitive Assessment; ADL: activities of daily living; VaMCI: vascular mild cognitive impairment.

In addition, significantly decreased amplitude of lowfrequency fluctuation (ALFF) in the left MTG had been reported in VaMCI [43]. In the present study, the left MTG was found to exhibit decreased FCS in the aMCI and VaMCI groups. Furthermore, compared with aMCI, the VaMCI group showed more serious FCS reduction in MTG. These results suggested that, as a critical network node with rich connections, the MTG was preferentially targeted in cognition impairment-related diseases, especially in the VaMCI group.

Subsequently, we made the left MTG-based wholebrain FC maps for further analysis. Notably, reduced FC between the left MTG and some frontal brain regions, including the right OFG and bilateral IFG, were mainly reported in the VaMCI group. Previous studies indicated that the small vessel disease-related cognitive impairments, including the deterioration of psychomotor speed, executive control, and global cognitive function, may be related to disruption and disconnection of the frontal-subcortical pathways [44]. Both IFG and OFG were associated with the executive function $[45,46]$. This is consistent with the notion that the dorsolateral prefrontal circuit is involved mainly in executive function [47, 48]. Using fMRI, decreased ALFF and FC in the frontal lobe have been repeatedly reported in VaMCI [49-52]. In addition, orbitofrontal syndromes, such as disinhibition and decreased social behavior, are more frequent in VaMCI than in aMCI. In the present study, disrupted FC between the frontal lobe and MTG was found in the MCI group, especially the VaMCI group. Furthermore, FC values between the left MTG and left IFG were associated with cognitive and living status, each reflected by the MoCA and ADL scores in VaMCI patients, which may be related to the executive dysfunction.

Several limitations need to be considered for this study. First, the sample size of the study was relatively small, and studies with a larger sample will be needed to replicate the current findings. Second, there were some subtle differences between the two MRI sequences. Unified MRI sequence parameters should be used in the future study.
In conclusion, the present study demonstrated for the first time that the MCI was associated with disrupted functional brain networks and significantly decreased FCS in the MTG. Furthermore, disrupted FC between the frontal lobe and MTG was found in the VaMCI group, which may be related to the executive dysfunction. These findings may open a novel way to better understand the pathophysiological mechanisms of MCI.

\section{Data Availability}

The datasets analyzed during the current study are not publicly available due to the unfinished study of the whole project but are available from the corresponding author on reasonable request.

\section{Conflicts of Interest}

The authors declare that they have no conflicts of interest.

\section{Authors' Contributions}

Hui Li and Shuai Gao carried out data collection and wrote the manuscript. Xiuqin Jia carried out data analysis. Tao Jiang and Kuncheng Li contributed to the conceptualization and design of the study and revised the manuscript. Hui Li and Shuai Gao contributed equally to this work.

\section{Acknowledgments}

This work was supported partly by grants from the National Natural Science Foundation of China (62076169) and Beijing Municipal Science and Technology Commission (Z171100000117001).

\section{Supplementary Materials}

The attached file of supplementary materials presents the results derived from one-sample $t$-tests of within-group analysis for the three groups. Figures S1-S3 show the functional nodes with a large number of connections in $\mathrm{HC}$, aMCI, 
and VaMCI, separately. Figures S4-S6 show the whole-brain FC of the left MTG in HC, aMCI, and VaMCI, separately. (Supplementary Materials)

\section{References}

[1] S. Gauthier, B. Reisberg, M. Zaudig et al., "Mild cognitive impairment," Lancet, vol. 367, no. 9518, pp. 1262-1270, 2006.

[2] P. Sachdev, R. Kalaria, J. O'Brien et al., "Diagnostic criteria for vascular cognitive disorders: a VASCOG statement," Alzheimer Disease and Associated Disorders, vol. 28, no. 3, pp. 206218, 2014.

[3] M. S. Albert, S. T. Dekosky, D. Dickson et al., "The diagnosis of mild cognitive impairment due to Alzheimer's disease: recommendations from the National Institute on Aging-Alzheimer's Association workgroups on diagnostic guidelines for Alzheimer's disease," Alzheimers \& Dementia, vol. 7, no. 3, pp. 270-279, 2011.

[4] N. L. Graham, T. Emery, and J. R. Hodges, "Distinctive cognitive profiles in Alzheimer's disease and subcortical vascular dementia," Journal of Neurology, Neurosurgery, and Psychiatry, vol. 75, no. 1, pp. 61-71, 2004.

[5] L. Yi, J. Wang, L. Jia et al., "Structural and functional changes in subcortical vascular mild cognitive impairment: a combined voxel-based morphometry and resting-state fMRI study," PLoS One, vol. 7, no. 9, 2012.

[6] Y. W. Sun, L. D. Qin, Y. Zhou et al., “Abnormal functional connectivity in patients with vascular cognitive impairment, no dementia: a resting-state functional magnetic resonance imaging study," Behavioural Brain Research, vol. 223, no. 2, pp. 388-394, 2011.

[7] D. Y. Lee, E. Fletcher, O. Martinez et al., "Vascular and degenerative processes differentially affect regional interhemispheric connections in normal aging, mild cognitive impairment, and Alzheimer disease," Stroke, vol. 41, no. 8, pp. 1791-1797, 2010.

[8] X. Li, D. Li, Q. Li et al., "Hippocampal subfield volumetry in patients with subcortical vascular mild cognitive impairment," Scientific Reports, vol. 6, no. 1, 2016.

[9] M. Pihlajamaki, A. M. Jauhiainen, and H. Soininen, "Structural and functional MRI in mild cognitive impairment," Current Alzheimer Research, vol. 6, no. 2, pp. 179-185, 2009.

[10] J. M. Papma, M. de Groot, I. de Koning et al., "Cerebral small vessel disease affects white matter microstructure in mild cognitive impairment," Human Brain Mapping, vol. 35, no. 6, pp. 2836-2851, 2014.

[11] B. Bosch, E. M. Arenaza-Urquijo, L. Rami et al., "Multiple DTI index analysis in normal aging, amnestic MCI and AD. Relationship with neuropsychological performance," Neurobiology of Aging, vol. 33, no. 1, pp. 61-74, 2012.

[12] D. Medina, L. Detoledo-Morrell, F. Urresta et al., "White matter changes in mild cognitive impairment and AD: a diffusion tensor imaging study," Neurobiology of Aging, vol. 27, no. 5, pp. 663-672, 2006.

[13] Y. Yu, X. Liang, H. Yu et al., "How does white matter microstructure differ between the vascular and amnestic mild cognitive impairment?," Oncotarget, vol. 8, no. 1, pp. 42-50, 2017.

[14] A. Lin, A. R. Laird, P. T. Fox, and J. H. Gao, "Multimodal MRI neuroimaging biomarkers for cognitive normal adults, amnestic mild cognitive impairment, and Alzheimer's disease," Neurology Research International, vol. 2012, Article ID 907409, 17 pages, 2012.
[15] L. Y. Yi, X. Liang, D. M. Liu et al., "Disrupted topological organization of resting-state functional brain network in subcortical vascular mild cognitive impairment," CNS Neuroscience \& Therapeutics, vol. 21, no. 10, pp. 846-854, 2015.

[16] E. Bullmore and O. Sporns, "Complex brain networks: graph theoretical analysis of structural and functional systems," Nature Reviews Neuroscience, vol. 10, no. 3, pp. 186-198, 2009.

[17] Y. He and A. Evans, "Graph theoretical modeling of brain connectivity," Current Opinion in Neurology, vol. 23, no. 4, pp. 341-350, 2010.

[18] Y. He, J. Wang, L. Wang et al., "Uncovering intrinsic modular organization of spontaneous brain activity in humans," PLoS One, vol. 4, no. 4, 2009.

[19] S. Achard, R. Salvador, B. Whitcher, J. Suckling, and E. Bullmore, "A resilient, low-frequency, small-world human brain functional network with highly connected association cortical hubs," Journal of Neuroscience, vol. 26, no. 1, pp. 6372, 2006.

[20] D. S. Bassett and E. T. Bullmore, "Small-world brain networks revisited," The Neuroscientist, vol. 23, no. 5, pp. 499-516, 2017.

[21] F. Liu, C. Zhu, Y. Wang et al., "Disrupted cortical hubs in functional brain networks in social anxiety disorder," Clinical Neurophysiology, vol. 126, no. 9, pp. 1711-1716, 2015.

[22] H. Li, X. Jia, Z. Qi et al., "Disrupted functional connectivity of cornu ammonis subregions in amnestic mild cognitive impairment: a longitudinal resting-state fMRI study," Frontiers in Human Neuroscience, vol. 12, 2018.

[23] R. C. Petersen, J. C. Stevens, M. Ganguli, E. G. Tangalos, J. L. Cummings, and S. T. DeKosky, "Practice parameter: early detection of dementia: mild cognitive impairment (an evidence-based review). Report of the Quality Standards Subcommittee of the American Academy of Neurology," Neurology, vol. 56, no. 9, pp. 1133-1142, 2001.

[24] P. Scheltens, L. J. Launer, F. Barkhof, H. C. Weinstein, and W. A. Gool, "Visual assessment of medial temporal lobe atrophy on magnetic resonance imaging: interobserver reliability," Journal of Neurology, vol. 242, no. 9, pp. 557-560, 1995.

[25] M. J. de Leon, J. Golomb, A. E. George et al., "The radiologic prediction of Alzheimer disease: the atrophic hippocampal formation," AJNR. American Journal of Neuroradiology, vol. 14, no. 4, pp. 897-906, 1993.

[26] American Psychiatric Association, Diagnostic and Statistical Manual of Mental Disorders, American Psychiatric Association, Arlington, 5th edition, 2013.

[27] Y. Chao-Gan and Z. Yu-Feng, "DPARSF: a MATLAB toolbox for "pipeline" data analysis of resting-state fMRI," Frontiers in Systems Neuroscience, vol. 4, no. 13, 2010.

[28] F. Liu, B. Xie, Y. Wang et al., "Characterization of posttraumatic stress disorder using resting-state fMRI with a multi-level parametric classification approach," Brain Topography, vol. 28, no. 2, pp. 221-237, 2015.

[29] Y. Behzadi, K. Restom, J. Liau, and T. T. Liu, “A component based noise correction method (CompCor) for BOLD and perfusion based fMRI," NeuroImage, vol. 37, no. 1, pp. 90-101, 2007.

[30] L. Wang, Z. Dai, H. Peng et al., "Overlapping and segregated resting-state functional connectivity in patients with major depressive disorder with and without childhood neglect," Human Brain Mapping, vol. 35, no. 4, pp. 1154-1166, 2014. 
[31] O. Sporns, C. J. Honey, and R. Kotter, "Identification and classification of hubs in brain networks," PLoS One, vol. 2, no. 10, 2007.

[32] O. Sporns, D. R. Chialvo, M. Kaiser, and C. C. Hilgetag, "Organization, development and function of complex brain networks," Trends in Cognitive Sciences, vol. 8, no. 9, pp. 418425, 2004.

[33] P. Hagmann, L. Cammoun, X. Gigandet et al., "Mapping the structural core of human cerebral cortex," PLoS Biology, vol. 6, no. 7, 2008.

[34] S. Gupta, J. C. Rajapakse, and R. E. Welsch, “Ambivert degree identifies crucial brain functional hubs and improves detection of Alzheimer's Disease and Autism Spectrum Disorder," NeuroImage: Clinical, vol. 25, article 102186, 2020.

[35] R. L. Buckner, J. Sepulcre, T. Talukdar et al., "Cortical hubs revealed by intrinsic functional connectivity: mapping, assessment of stability, and relation to Alzheimer's disease," Journal of Neuroscience, vol. 29, no. 6, pp. 1860-1873, 2009.

[36] T. Raettig and S. A. Kotz, "Auditory processing of different types of pseudo-words: an event-related fMRI study," NeuroImage, vol. 39, no. 3, pp. 1420-1428, 2008.

[37] B. R. Reed, J. L. Eberling, D. Mungas, M. Weiner, J. H. Kramer, and W. J. Jagust, "Effects of white matter lesions and lacunes on cortical function," Archives of Neurology, vol. 61, no. 10, pp. 1545-1550, 2004.

[38] M. Grau-Olivares, D. Bartrés-Faz, A. Arboix et al., "Mild cognitive impairment after lacunar infarction: voxel-based morphometry and neuropsychological assessment," Cerebrovascular Diseases, vol. 23, no. 5-6, pp. 353-361, 2007.

[39] G. T. Stebbins, D. L. Nyenhuis, C. Wang et al., "Gray matter atrophy in patients with ischemic stroke with cognitive impairment," Stroke, vol. 39, no. 3, pp. 785-793, 2008.

[40] K. Li, W. Chan, R. S. Doody, J. Quinn, and S. Luo, "Prediction of conversion to Alzheimer's disease with longitudinal measures and time-to-event data," Journal of Alzheimers Disease, vol. 58, no. 2, pp. 361-371, 2017.

[41] C. Xue, B. Yuan, Y. Yue et al., "Distinct Disruptive Patterns of Default Mode Subnetwork Connectivity Across the Spectrum of Preclinical Alzheimer's Disease," Frontiers in Aging Neuroscience, vol. 11, p. 307, 2019.

[42] M. Li, G. Zheng, Y. Zheng et al., "Alterations in resting-state functional connectivity of the default mode network in amnestic mild cognitive impairment: an fMRI study," BMC Medical Imaging, vol. 17, no. 1, p. 48, 2017.

[43] C. Li, J. Yang, X. Yin et al., “Abnormal intrinsic brain activity patterns in leukoaraiosis with and without cognitive impairment," Behavioural Brain Research, vol. 292, pp. 409-413, 2015.

[44] J. A. Pettersen, G. Sathiyamoorthy, F. Q. Gao et al., "Microbleed topography, leukoaraiosis, and cognition in probable Alzheimer disease from the Sunnybrook Dementia Study," Archives of Neurology, vol. 65, no. 6, pp. 790-795, 2008.

[45] L. Garcia-Alvarez, J. J. Gomar, A. Sousa, M. P. Garcia-Portilla, and T. E. Goldberg, "Breadth and depth of working memory and executive function compromises in mild cognitive impairment and their relationships to frontal lobe morphometry and functional competence," Alzheimer's \& Dementia: Diagnosis, Assessment \& Disease Monitoring, vol. 11, no. 1, pp. 170-179, 2019.

[46] E. de Guise, A. Y. Alturki, M. Lague-Beauvais et al., "Olfactory and executive dysfunctions following orbito-basal lesions in traumatic brain injury," Brain Injury, vol. 29, no. 6, pp. 730$738,2015$.

[47] J. M. Unterrainer and A. M. Owen, "Planning and problem solving: from neuropsychology to functional neuroimaging," Journal of Physiology-Paris, vol. 99, no. 4-6, pp. 308-317, 2006.

[48] R. M. Bonelli and J. L. Cummings, "Frontal-subcortical circuitry and behavior," Dialogues in Clinical Neuroscience, vol. 9, no. 2, pp. 141-151, 2007.

[49] Y. Zhuang, Y. Shi, J. Zhang et al., "Neurologic Factors in Patients with Vascular Mild Cognitive Impairment Based on fMRI," World Neurosurgery, vol. 149, pp. 461-469, 2011.

[50] M. Zuo, Y. Xu, X. Zhang et al., “Aberrant brain regional homogeneity and functional connectivity of entorhinal cortex in vascular mild cognitive impairment: a resting-state functional MRI study," Frontiers in Neurology, vol. 9, no. 1177, 2018.

[51] X. Zhou, X. Hu, C. Zhang et al., "Aberrant functional connectivity and structural atrophy in subcortical vascular cognitive impairment: relationship with cognitive impairments," Frontiers in Aging Neuroscience, vol. 8, no. 14, 2016.

[52] L. Ni, R. Liu, Z. Yin et al., “Aberrant spontaneous brain activity in patients with mild cognitive impairment and concomitant lacunar infarction: a resting-state functional MRI study," Journal of Alzheimer's Disease, vol. 50, no. 4, pp. 1243-1254, 2016. 